\title{
Evaluación de las propiedades del subsuelo de Badajoz (España) con fines constructivos mediante Sistemas de Información Geográfica
}

\author{
Evaluation of subsoil properties of Badajoz (Spain) for construction \\ purposes using Geographic Information Systems \\ $\underline{\text { F. Hipólito-Ojalvo }}^{(*)}$, F. Zamora-Polo ${ }^{(*)(* *)}$, A. Luque $^{(* *)}$, I. Naharro-Sequeda ${ }^{(*)}$
}

\section{RESUMEN}

El estudio de las propiedades del subsuelo resulta fundamental para el diseño de las cimentaciones y para prevenir futuras patologías estructurales. En este trabajo se analiza la información procedente de 459 sondeos del subsuelo de Badajoz. Se describe la generación de un banco de datos así como la elaboración de un conjunto de planos que constituyen el Mapa Geotécnico Básico de Badajoz. Están recogidas, así mismo, las características más representativas en cada estrato. Se han identificado ocho capas representativas del corte estratigráfico de la ciudad. La cartografía geotécnica ha sido obtenida gráficamente mediante isolíneas, indicando la posición y los espesores de las estratigrafías. El estudio puede resultar de interés tanto para técnicos (ingenieros y arquitectos) como para las autoridades con competencias en el ámbito urbanístico.

Palabras clave: Cartografía geotécnica, SIG, cimentaciones, propiedades del suelo.

\section{ABSTRACT}

Subsoil properties study is crucial in order to design foundations and to prevent structural pathologies. In this paper, 459 explorations of Badajoz subsoil were analyzed; they were carried out during two decades. A bank of data creation was described, as well as the elaboration of a set of maps. They constitute the Basic Geotechnical Map of Badajoz. In these maps, the most representative characteristics of each stratum are also collected from a building perspective. In the study eight representative stratums were found. The geotechnical cartography has been obtained graphically by isolines, indicating the position and thickness of the stratums mentioned above. The study could be of interest both to technicians competent in the field of building and urban planning.

Keywords: Geotechnical Cartography, GIS, Foundations, Soil properties.

(*) Universidad de Extremadura. Departamento de Ingeniería Mecánica, Energética y de los Materiales.

Escuela de Ingenierías Industriales, Badajoz (España).

${ }^{(* *)}$ Universidad de Sevilla. Departamento de Ingeniería del Diseño. Escuela Politécnica Superior. Sevilla (España). Persona de contacto/Corresponding author: fhipolito@unex.es (F. Hipólito-Ojalvo).

ORCID: https://orcid.org/oooo-0003-2996-9731 (F. Hipólito-Ojalvo); https://orcid.org/oooo-0002-9700-6809 (F. Zamora-Polo); https://orcid.org/oooo-0002-1205-4722 (A. Luque); https://orcid.org/oooo-0002-5934-2967 (I. Naharro-Sequeda).

Cómo citar este artículo/Citation: Hipólito-Ojalvo, F.; Zamora-Polo, F.; Luque, A.; Naharro-Sequeda, I. (2019). Evaluación de las propiedades del subsuelo de Badajoz (España) con fines constructivos mediante Sistemas de Información Geográfica. Informes de la Construcción, 71(556): e309. https://doi.org/10.3989/ic.65204.

Copyright: (C) 2019 CSIC. Este es un artículo de acceso abierto distribuido bajo los términos de la licencia de uso y distribución Creative Commons Reconocimiento 4.o Internacional (CC BY 4.0). 


\section{INTRODUCCIÓN}

El conocimiento de las propiedades del subsuelo resulta esencial para el diseño adecuado de las cimentaciones e infraestructuras civiles, así como para prevenir posibles patologías estructurales $(1,2)$. Del mismo modo, el conocimiento de las características del terreno es una herramienta fundamental para el desarrollo de los planes urbanísticos (3). En este sentido, muchas ciudades crecen de forma incontrolada en condiciones adversas poniendo en riesgo la integridad estructural de los futuros edificios. Por ello, un conocimiento más profundo de las propiedades del suelo por parte de técnicos, políticos y otros actores del sector de la construcción puede suponer un avance en la eficiencia de éste. Los Sistemas de Información Geográfica (SIG) permiten el almacenaje, el análisis y la recuperación de información previamente obtenida y geográficamente referenciada.

Así mismo, los SIG facilitan la confección de mapas que posibilitan el análisis de los resultados $(4,5)$, siendo utilizados en diversas aplicaciones como pueden ser los usos energéticos $(6,7,8)$, medioambientales $(9,10,11)$, en agricultura $(12,13)$, para fines meteorológicos $(14,15)$, etc.

En el ámbito de la Mecánica del Suelo, los Sistemas de Información Geográfica han sido previamente aplicados en: la respuesta de los suelos de Barcelona ante un terremoto (16), el estudio de suelos expansivo $(1,5)$, el uso del suelo para una aplicación particular como pudiera ser una mina (17), un túnel (18), o el análisis de estabilidad de taludes (19).

En España, distintos autores realizaron mapas geotécnicos de ciudades como Granada (20), Murcia (21), Sevilla (22) y Melilla (23). Sin embargo, los estudios han estado centrados en otros objetivos distintos a los de este trabajo, como pueden ser los movimientos sísmicos (Granada), el análisis de la subsidencia (Murcia), la inspección técnica de edificios (Sevilla) y la influencia del mar (Melilla). Hasta donde sabemos, ningún trabajo ha abordado previamente el desarrollo de la cartografía geotécnica de la ciudad de Badajoz. Badajoz es una ciudad española, localizada cerca de la frontera con Portugal. Su población en 2017 ascendió a 150.454 habitantes (24) y su extensión es de $1470,43 \mathrm{~km}^{2}$ (25). Está atravesada por el río Guadiana que la divide en dos zonas claramente diferenciadas.

Los principales objetivos de este trabajo son:

- Crear una base de datos geoespacial con los resultados de los estudios geotécnicos realizados en la ciudad de Badajoz entre 1990 y 2010.

- Desarrollar una cartografía geotécnica mediante la utilización de SIG que permita identificar las mejores áreas para construir, así como predecir posibles problemas de patologías estructurales.

Además de la introducción inicial, el trabajo se presenta en cuatro secciones y su estructura es la siguiente: en la sección segunda se describen los materiales y métodos experimentales, en la tercera sección se presentan los resultados y la discusión de los mismos utilizando para ello técnicas geoestadísticas.

\section{MATERIALES Y MÉTODOS EXPERIMENTALES}

En este trabajo se analizan 459 sondeos procedentes de 387 estudios geotécnicos. Estos estudios fueron realizados por dos laboratorios homologados de las compañías: LYCCSA, y ELABOREX entre los años 1990 y 2010. Los estudios incluyen los siguientes ensayos normalizados: límites de Atterberg (Límite Líquido e Índice de Plasticidad) (26), análisis granulométrico por tamizado (27), ensayo de compresión simple (28), SPT (29) y DPSH $(30,31)$. La localización de todos los estudios geotécnicos se muestra en la Figura 1 (32).

Los resultados obtenidos fueron almacenados en una ficha de recogida de datos que se especificará en la siguiente sección. Éstos se procesaron utilizando Microsoft Excel(C), que facilita el acopio masivo de valores mediante la selección de filtros, relacionando gran cantidad de datos de cada estrato de forma independiente. Así quedan fácilmente accesibles las características de techo y espesor de las capas para su posterior tratamiento en el SIG. Se ha creado una base de datos en formato MySQLC, permitiendo con ello, consultas relativas a los análisis geotécnicos introduciendo cualquier indicador de posicionamiento conocido. Para la obtención de los mapas geotécnicos fue utilizada la herramienta informática GIS SURFER(C) (versión 9). GIS SURFER (C) permite obtener en un entorno de Windows (C) la cartografía geotécnica. Está basado en la creación de cuadrículas que posibilitan una representación gráfica de las variables incluyendo imágenes de contorno, sombras, colores, etc.

La Figura 2 muestra el procedimiento para el análisis geoestadístico de los datos (33).

El mapa inicial desde el cual se parte para el desarrollo del estudio fue proporcionado por la Dirección General de Urbanismo y Ordenación del Territorio de la Junta de Extremadura. Posteriormente se procedió a su manipulación fraccionándolo en manzanas en base a un plano de Plan General Municipal de Badajoz y los datos gráficos proporcionados por la Dirección General del Catastro del Ministerio de Hacienda y Administraciones Públicas. La cuadrícula elegida mide 70,6m x 70,2m porque, con estas dimensiones, el estudio se ajustaba a un cálculo con mayor precisión de las curvas de nivel. Las medidas de la cuadrícula han sido obtenidas tras desechar las urbanizaciones más alejadas del núcleo urbano consolidado, por proporcionar valores faltos de homogeneidad. Dichas dimensiones garantizan un número de sondeos óptimo para el posterior tratamiento mediante $S I G$.

\subsection{Interpolación geoestadística}

En geoestadística, se utiliza la variable regionalizada definida como una función que tiene un valor definido en cada punto del espacio y vigencia en un tiempo concreto. En nuestro caso, un ejemplo de variables regionalizadas son los parámetros denominados techo y espesor de las capas geotécnicas.

El variograma, también conocido como semi-variograma, es una herramienta central en el estudio geoestadístico. Intenta capturar el grado de discontinuidad de una función midiendo el grado de correlación o dependencia entre dos pares de muestras separadas una distancia $(h)$ en una dirección determinada (34).

El procedimiento de interpolación seguido en este trabajo se ajusta a lo propuesto por Bosque (35). En primer lugar se calcula el variograma y hallamos una función que se ajusta al variograma: $g(h)$. Esta función puede ser exponencial o lineal. Los pesos $\left(W_{i j}\right)$ pueden ser obtenidos del variograma teórico, conforme a lo propuesto por Matheron en su teoría de las variables regionalizadas (36). 


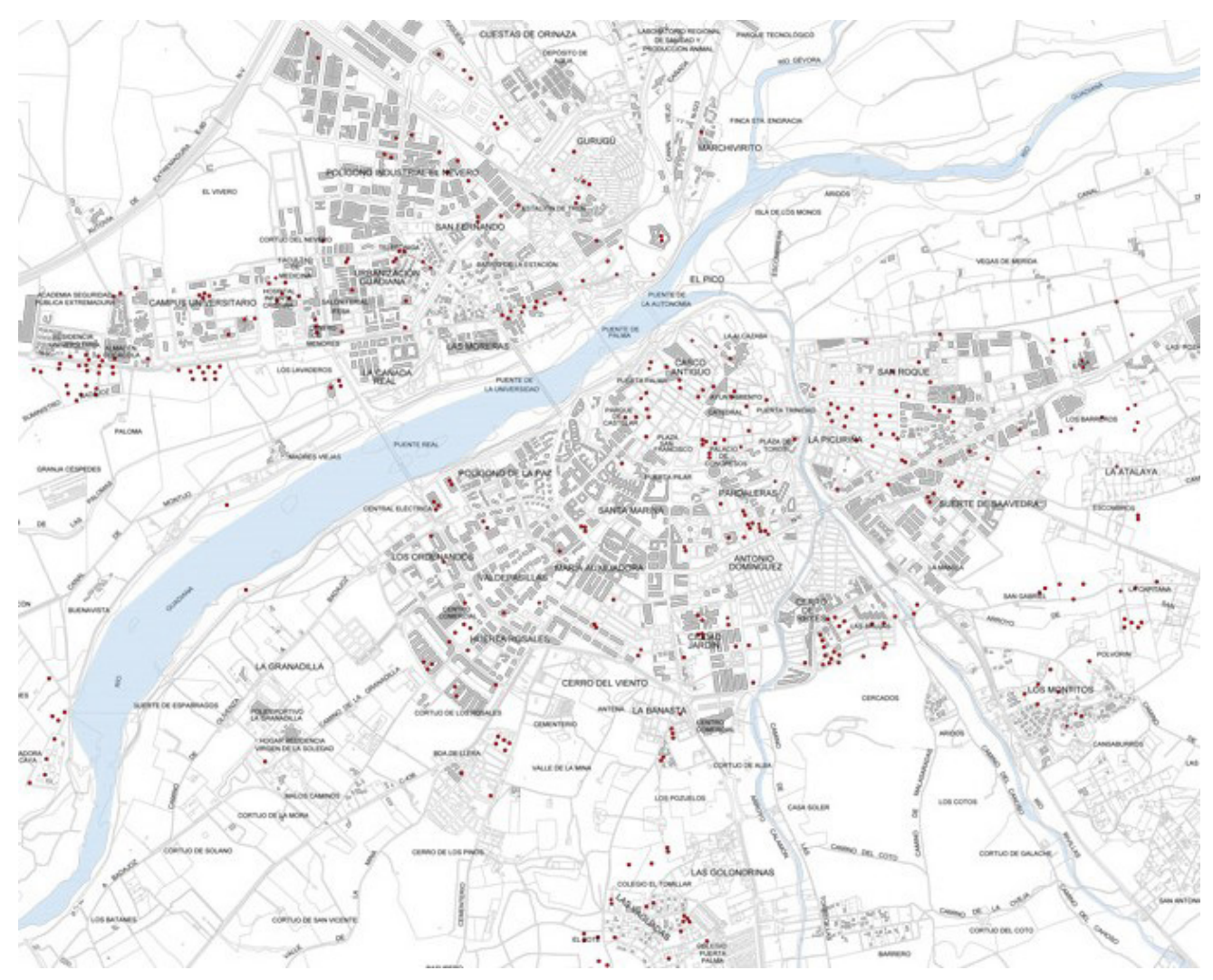

Figura 1. Localización de los estudios geotécnicos (32).

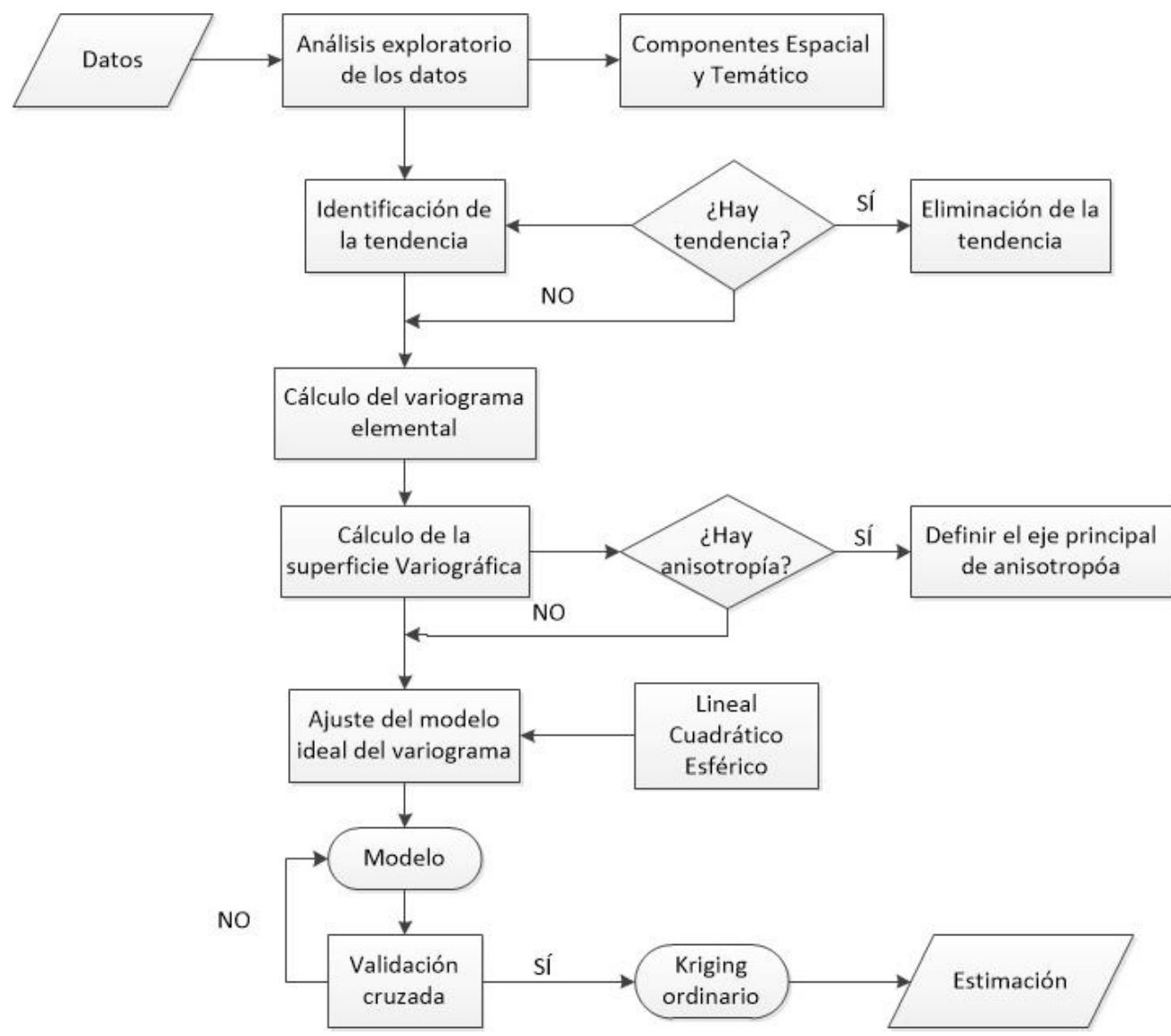

Figura 2. Procedimiento de análisis geoestadístico basado en Pintos et al. (33). 
La interpolación espacial realizada con el kriging se obtiene mediante una combinación lineal ponderada de los valores de la altura. Dicho método provee, a partir de un conjunto de puntos regular o irregularmente distribuidos, valores estimados de lugares donde no hay información, es decir, estima la variable en los puntos no muestrales considerando la estructura de correlación espacial seleccionada e integrando la información obtenida de forma directa en los puntos de la muestra.

\section{RESULTADOS Y DISCUSIÓN}

\subsection{Creación de una base de datos estadística}

El primer paso de la investigación fue crear una base de datos donde se incorporarán todos los parámetros de los estudios geotécnicos realizados. En cada uno de éstos se contaba con la siguiente información:

- Datos generales: promotor, laboratorio, fecha del estudio y ubicación física (dirección).

- Un mapa con la localización del sondeo.

- Coordenadas GPS del sondeo.

- Resultados: Límite líquido conforme al método de Atterberg's $\left(w_{L}\right)$, índice de plasticidad $\left(I_{p}\right)$, porcentaje que pasa a través del tamiz 200 ASTM $\left(T_{200}\right)$, resistencia en el ensayo de compresión simple $\left(q_{u}\right)$, número de golpes requeridos para alcanzar $30 \mathrm{~cm}$ en el ensayo de penetración estándar o en el ensayo DPSH $(N)$.

- Profundidad de corte del nivel freático.
- Recomendaciones para la cimentación.

- Conclusiones.

- Otros comentarios.

La Figura 3 muestra un ejemplo con la hoja de recogida de datos utilizada en el estudio. Estos archivos pueden ser consultados en la Tesis Doctoral de uno de los autores del trabajo (32).

Se han examinado las características del suelo urbano en el tramo cronológico 1990-2010 (Figura 4) por ser las dos décadas de mayor solicitud de estudios geotécnicos. Una demanda propiciada por el impulso inmobiliario en los años de cre-

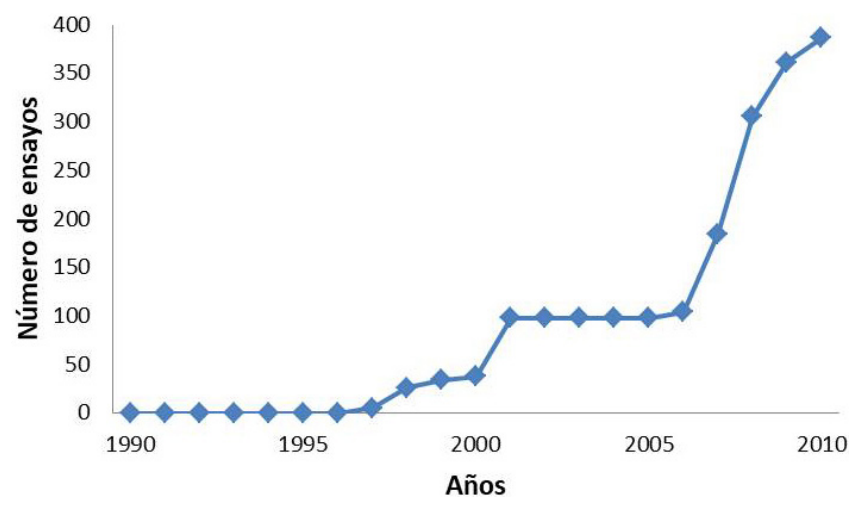

Figura 4. Distribución de los estudios en función del tiempo (32).

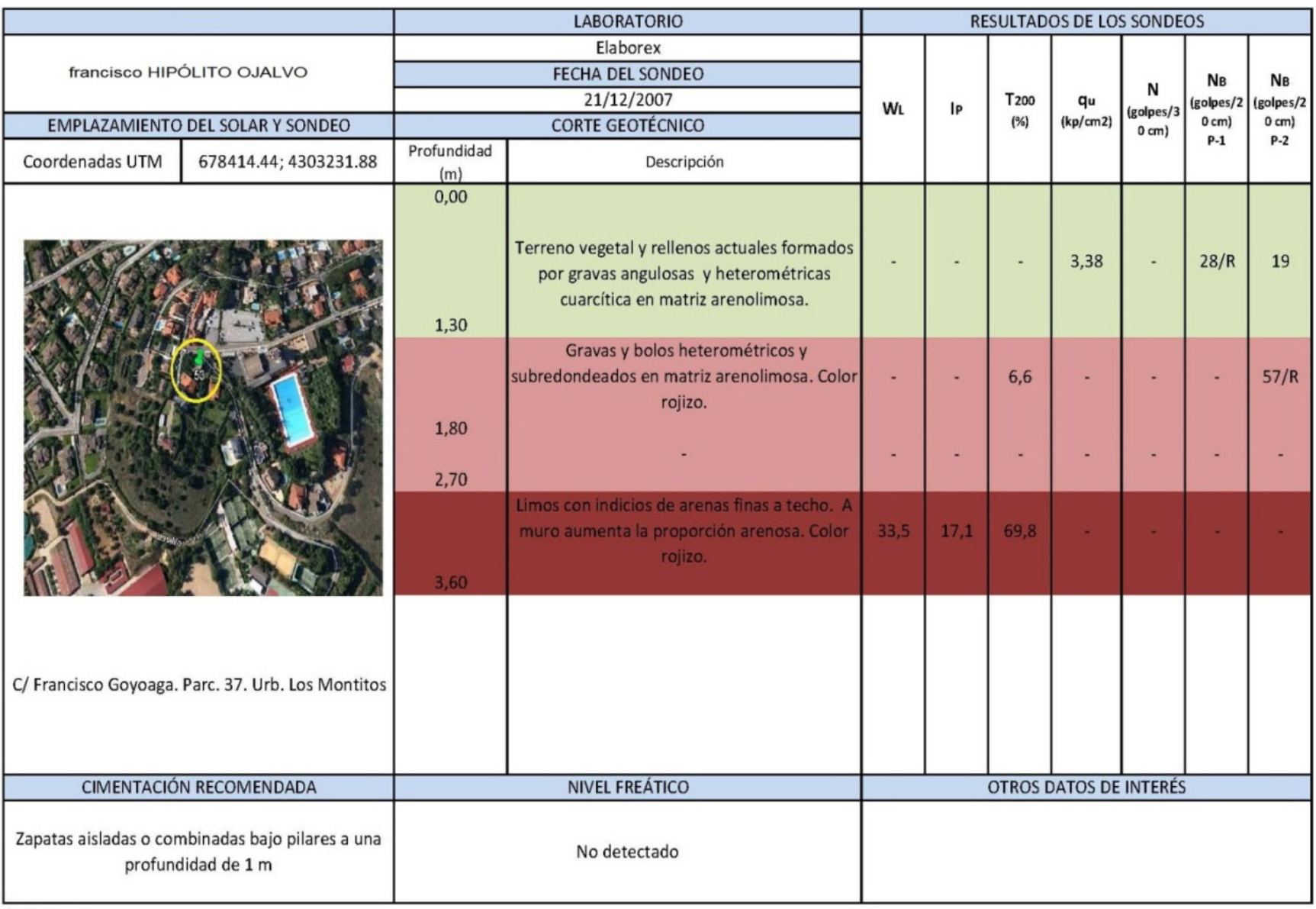


cimiento económico, por la recepción de Fondos Europeos, (Badajoz es Zona Objetivo I), y por la entrada en vigor del Código Técnico de la Edificación (37).

En los análisis geotécnicos, un número mínimo de estudios son requeridos. De esta forma Hernández et al. (23) distinguen tres zonas:

a) Zona de espera: Cronológicamente es la etapa inicial, el número de ensayos crece monótonamente con velocidad lenta a lo largo del tiempo.

b) Zona abierta: Presenta un incremento lineal de los ensayos a lo largo del tiempo. Se observa un incremento considerable en el número de estudios. En esta zona se recomienda realizar los ensayos geoestadísticos y el desarrollo de los mapas de variables regionalizadas.

c) Zona cerrada: La velocidad decrece monótonamente, y tener más ensayos no proporciona gran ayuda de cara a la realización de los estudios geotécnicos.

Como se puede observar en la Figura 4, con los estudios realizados se ha alcanzado la zona abierta, siendo el momento óptimo para el desarrollo del estudio geoestadístico de las propiedades geotécnicas de la ciudad de Badajoz.

\subsection{Densidad de los datos y distribución}

La fiabilidad de los mapas generados en el análisis geoestadístico va a depender de dos variables: la densidad y la distribución de los datos.

La densidad es definida como la relación entre el número de sondeos $(s)$ sobre el área estudiada $(A)$ expresada en sondeos por kilómetro cuadrado $\left(\mathrm{s} / \mathrm{km}^{2}\right)$. En este trabajo se ha considerado un área de la ciudad de Badajoz de $36,25 \mathrm{~km}^{2}$, siendo ésta el área más representativa desde el punto de vista urbanístico. La densidad media de información en función del número de sondeos con los que se trabaja es de $10,7 \mathrm{~s} / \mathrm{km}^{2}$. Este número es más alto que otros estudios publicados en la bibliografía $\left(6,5 \mathrm{~s} / \mathrm{km}^{2}\right.$ (23) y $\left.3,4 \mathrm{~s} / \mathrm{km}^{2}(22)\right)$.

Otro factor importante que debe ser considerado es la distribución de información. La disposición de sondeos debe ser lo más homogénea posible. Como se puede observar en la Figura 1, la distribución de los sondeos no es completamente homogénea. Esto es debido a que los puntos de interés de los estudios geotécnicos corresponden a la localización de futuras construcciones.

Para estudiar analíticamente la distribución de las exploraciones (20) se debe verificar que la distancia entre unidades básicas es menor que la media de las distancias obtenidas mediante la siguiente ecuación:

$$
\mathrm{d}(\mathrm{m})=100 \sqrt{E}
$$

Donde $E$ es la media de las exploraciones por $\mathrm{km}^{2}\left(10,7 \mathrm{~s} / \mathrm{km}^{2}\right.$ en nuestro estudio). De esta forma,

$$
\mathrm{d}(\mathrm{m})=327 \mathrm{~m}
$$

El valor anterior se corresponde con la distancia máxima que debe haber entre dos unidades básicas de información para tener la certeza de poder construir unas curvas de nivel fiables por el método de interpolación.

\subsection{Análisis estratigráfico}

A partir de los resultados de exploración, se puede concluir que el suelo de la ciudad de Badajoz está compuesto por ocho tipos estratigráficos: estrato rocoso, gravas, gravas en arenas, arena, gravas en arcilla, arenas en arcilla, arcilla y limos, y rellenos.

En la capa superior se encuentran los rellenos con una baja capacidad portante. Posteriormente se puede encontrar una capa de arcillas y limos que tiene un comportamiento hidrogeológico capaz de alojar gran cantidad de agua, pero la transmite con dificultad. A continuación, se suelen encontrar intercaladas: arenas en arcillas y gravas en arcillas. En estas zonas es frecuente localizar las arcillas expansivas, capaces de provocar patologías a causa de su hinchamiento. Por debajo se esta capa se confina un estrato arenoso y otro de transición (arenas y gravas), limitados inferiormente por una franja de gravas, sumamente estable para las cimentaciones.

En la cota inferior del perfil estratigráfico se localiza el estrato rocoso compuesto por materiales con una alta resistencia a la compresión y es ideal para las cimentaciones usando zapatas.

El nivel freático constituye un área no consolidada del terreno en el cual aparece agua. Es muy importante para el diseño y construcción de las cimentaciones. Su posición es variable y depende tanto de condiciones geológicas como de condiciones climatológicas.

Obviamente el perfil estratigráfico varía de unas zonas a otras. De esta forma, en unas áreas pueden no existir algunas capas bien por factores hidrogeológicos o debido a alteraciones inducidas por factores humanos.

La Tabla 1 muestra los resultados experimentales referentes a las diversas capas estratigráficas obtenidas en los sondeos del estudio.

Mediante la utilización de técnicas geoestadísticas (método Kriging), se pueden obtener los mapas de igual profundidad e igual espesor.

Las líneas de igual profundidad se definen como aquellas en las cuales todos los puntos que la definen tienen la misma cota. Se corresponden por tanto con el "techo" de la capa a la que hace referencia, entendiéndose como tal la de mayor altura y coincide con el fondo de la estratigrafía de la capa inmediatamente superior. Son conocidas también como líneas isobáticas o isobatas. Las líneas de igual espesor son aquellas que su distancia entre el techo y el fondo de la capa es el mismo.

Se han realizado los mapas con líneas de igual profundidad e igual espesor para todas las capas del terreno. Las Figuras 5 y 6 muestran los mapas con las líneas de igual profundidad y espesor para el estrato rocoso. Un tratamiento similar pudiera hacerse para el resto de las capas.

Una forma distinta de expresar la información anterior es mediante la utilización de mapas tridimensionales. En la Figura 7 se muestra una representación tridimensional de la profundidad del estrato rocoso en la ciudad de Badajoz. 
Tabla 1. Promedio de las propiedades geotécnicas para cada una de las capas estratigráficas (32)

\begin{tabular}{|c|c|c|c|c|c|c|c|c|}
\hline \multirow[b]{2}{*}{$\begin{array}{l}\text { Parámetros } \\
\text { geotécnicos }\end{array}$} & \multicolumn{8}{|c|}{ Capa } \\
\hline & Rellenos & $\begin{array}{l}\text { Limos y } \\
\text { arcillas }\end{array}$ & $\begin{array}{c}\text { Arenas en } \\
\text { arcillas }\end{array}$ & $\begin{array}{c}\text { Gravas en } \\
\text { arenas }\end{array}$ & Arena & $\begin{array}{c}\text { Grava en } \\
\text { arena }\end{array}$ & Grava & $\begin{array}{l}\text { Estrato } \\
\text { rocoso }\end{array}$ \\
\hline $\begin{array}{l}\text { Número de sondeos con } \\
\text { esta capa }\end{array}$ & 435 & 239 & 333 & 43 & 81 & 79 & 106 & 112 \\
\hline Profundidad [m] & & 2.16 & 1.84 & 1.67 & 2.81 & 2.98 & 1.89 & 4.97 \\
\hline Espesor [m] & 0.86 & 2.02 & 2.07 & 1.54 & 2.12 & 2.11 & 2.14 & 3.10 \\
\hline $\begin{array}{l}\text { Resistencia a la } \\
\text { compresión }\left[\mathrm{kp} / \mathrm{cm}^{2}\right]\end{array}$ & 1.93 & 1.95 & 1.83 & 1.95 & 2.12 & 2.18 & 2.725 & 9.93 \\
\hline NSPT & 12 & $>25$ & $>20$ & $>19$ & $>25$ & 28 & 29 & $>30$ \\
\hline NDPSH & 13 & $>20$ & $>18$ & 21 & 24 & 34 & $>15$ & $>25$ \\
\hline Porcentaje de finos & 68.2 & 65.91 & 57.4 & 46 & 52.65 & 38.28 & 24.38 & 53.88 \\
\hline Límite Líquido (\%) & 32.35 & 40.91 & 39.45 & 42.36 & 32.85 & 37.72 & 33.19 & 40.44 \\
\hline Índice Plástico (\%) & 13.84 & 18.71 & 17.48 & 20.88 & 14.57 & 17.74 & 15.52 & 17.95 \\
\hline $\begin{array}{l}\text { Clasificación de acuerdo a } \\
\text { Casagrande }\end{array}$ & $\mathrm{CL}$ & $\mathrm{CL}$ & $\mathrm{SC}$ & GC-MH-OH & $\mathrm{SC}$ & GC & GC & SC-GC \\
\hline
\end{tabular}

El estrato rocoso está compuesto por suelo residual procedente de piedra granodiorita, y en sus tramos menos alterados, se originan gravas y arenas limosas de litología ígnea. Cohabitan con zonas de granito de meteorización VI y V, anfibolitas verdosas y venas de cuarzo con pátinas de oxidación. También se contemplan pizarras. Este estrato suele ser limítrofe con el muro de gravas con arcillas.

La capacidad portante del estrato rocoso es muy alta y resulta un buen plano de apoyo para la cimentación. Presenta una excelente resistencia a la compresión y es un material sobresaliente siempre que las rocas no presenten fisuras o estén estratificadas.
La cimentación que se suele recomendar es de tipología superficial, preferentemente zapatas. Constituye la forma de ejecución más económica y rápida, presentando la ventaja de minimizar la existencia de asientos diferenciales.

Como se muestra en la Tabla 1, se ha encontrado este estrato en 112 sondeos, de los cuales presentan rechazo en 42 de ellos en el ensayo de penetración estándar y en 44 en el ensayo de penetración dinámica.

Su espesor máximo es de $14 m$ y la profundidad mayor asciende a $-16,5 \mathrm{~m}$. El espesor medio registrado es de 3,1m (este va-

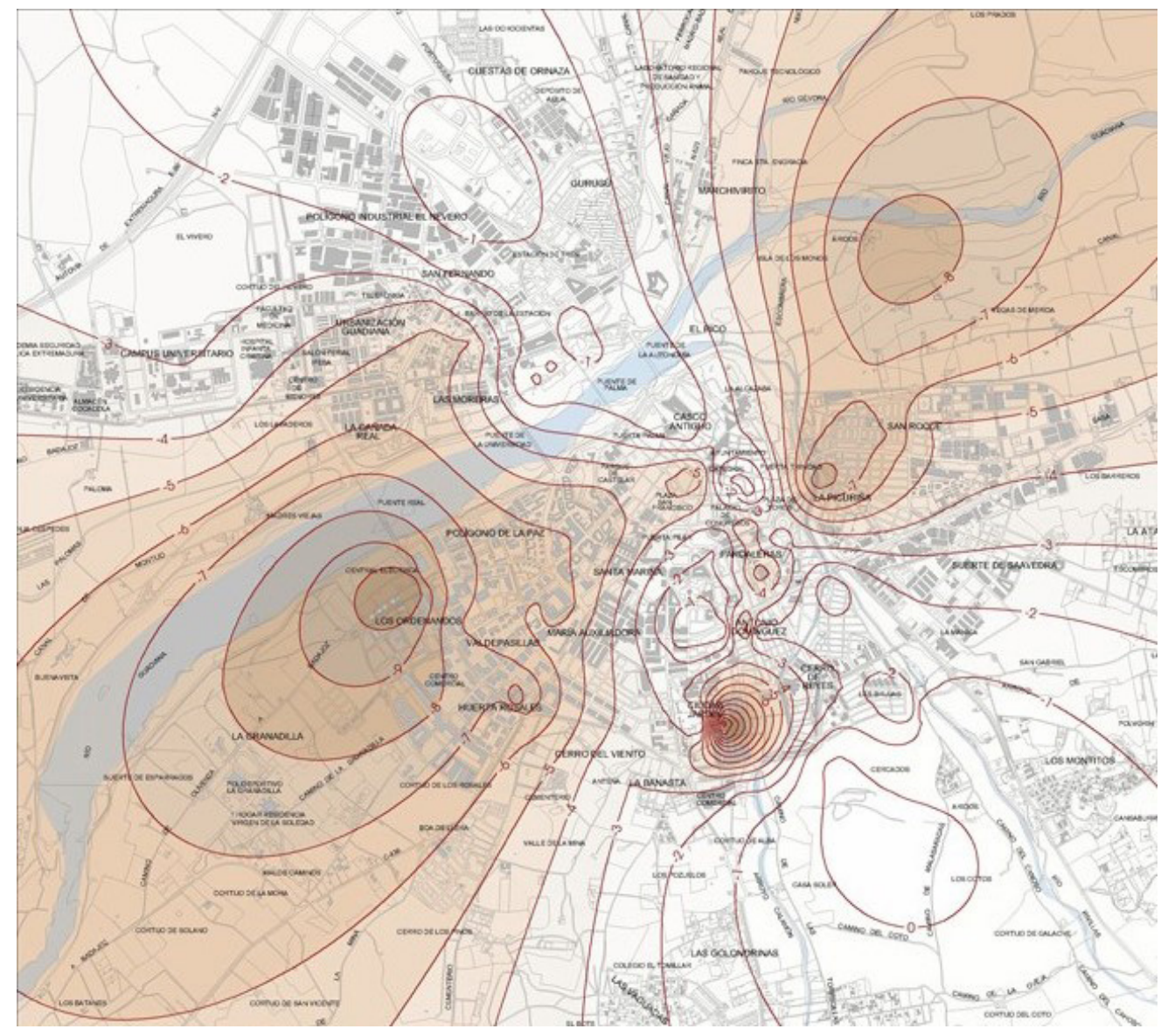

Figura 5. Líneas de igual profundidad del estrato rocoso en la ciudad de Badajoz [m] (32). 
lor se ha obtenido en ensayos que no presentan rechazo en el ensayo de penetración).

La resistencia a la compresión simple refleja un valor medio de $9,93 \mathrm{kp} / \mathrm{cm}^{2}$. Su capacidad resistente se ajusta a valores propicios para emplazar el plano de cimentación.

Respecto al resto de las capas, conviene destacar que el relleno superficial de Badajoz está compuesto por material de naturaleza antrópica, arenas limosas mezcladas con gravas y bolos, restos cerámicos y materia orgánica.

Se aprecia un estrato de arcillas y limos considerable, sobre todo en los depósitos aluviales, donde la variabilidad del nivel freático presenta mayor fluctuación. Surgen entonces arcillas marrón grisáceas de alta plasticidad con brillos sedosos.

Repartida por todo el casco urbano está extendida la estratigrafía de intercalación de arenas en arcillas. Esto mismo sucede con la capa de gravas en arcillas. Se ha detectado que es sumamente homogénea y de débil cementación.

Las franjas de gravas en arenas y gravas, constituyen un material resistente muy deseado por los técnicos para emplazar la cimentación.

Sin duda, la información proporcionada en los mapas de las Figuras 6 y 7 aportan información relevante para el conoci-

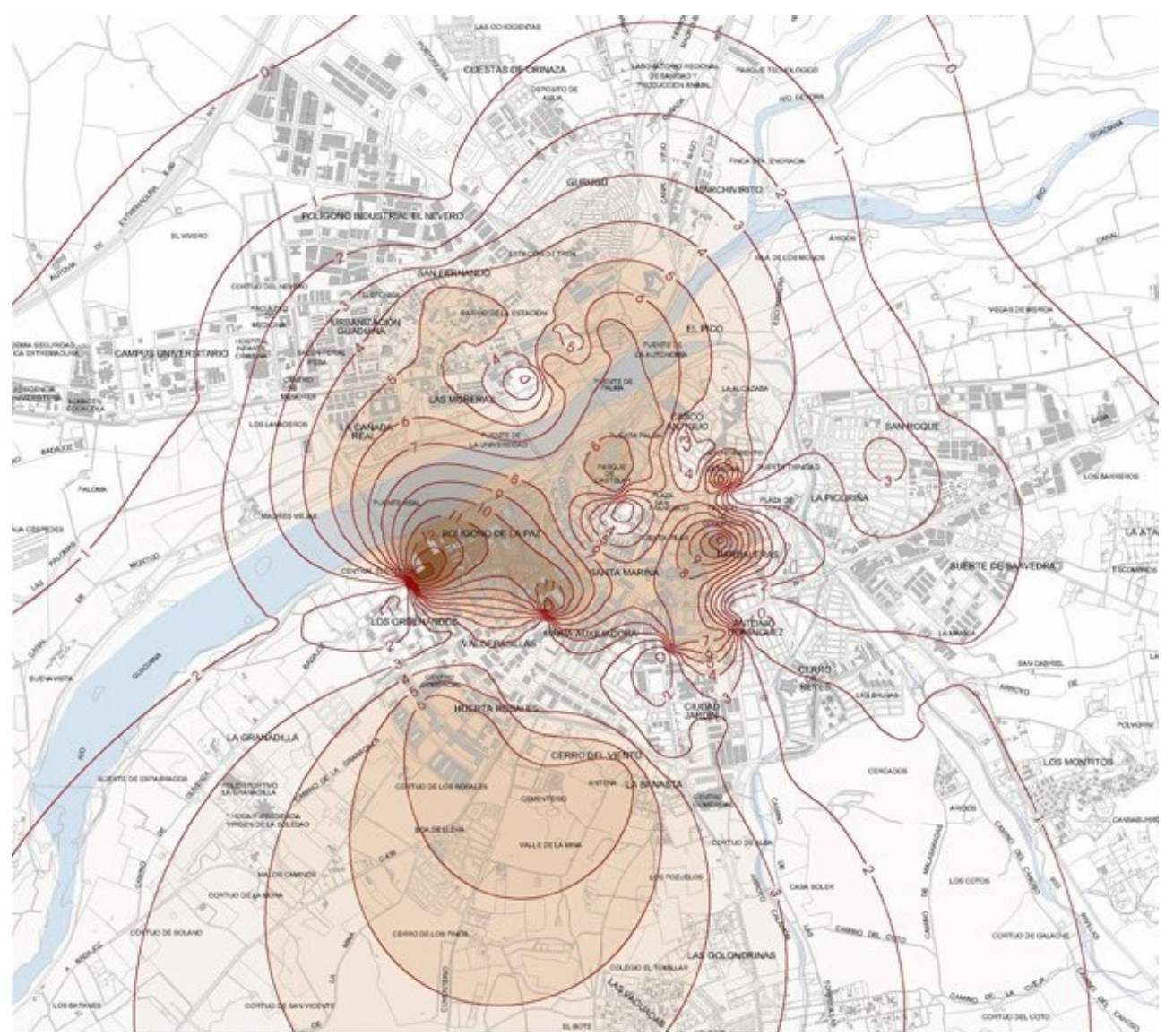

Figura 6. Líneas de igual espesor del estrato rocoso en la ciudad de Badajoz [m] (32).

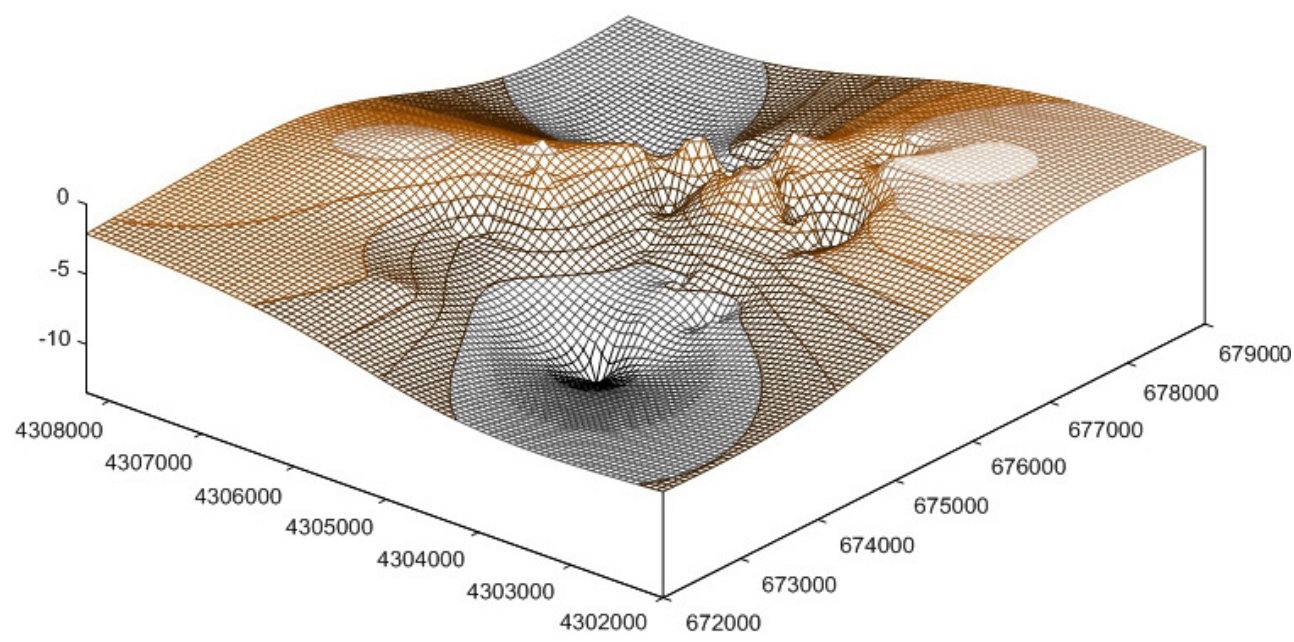

Figura 7. Representación tridimensional de la profundidad del estrato rocoso [m] (32). 
miento de las áreas urbanas. Así mismo, facilitan la redacción de los proyectos, la elección de la cimentación, y ayudan a prevenir futuras patologías estructurales (1).

\section{CONCLUSIONES}

El presente estudio ofrece a lugareños, técnicos y políticos un documento básico que optimiza la ordenación territorial y evalúa la cimentación de los inmuebles. Proporciona, así mismo, una herramienta gráfica para tomar decisiones urbanísticas, pone en valor las particularidades del parcelario y orienta al proyectar la cimentación.

Ha sido confeccionado un conjunto de planos que constituyen el Mapa Geotécnico Básico de Badajoz, recogiendo las propiedades de sus estratos y su aplicación a la edificación. Tras confeccionar un banco de datos con 459 sondeos, se identifican en el casco urbano nueve capas geotécnicas: rellenos, arcillas y limos, arenas en arcillas, gravas en arcillas, arenas, gravas en arenas, gravas, el estrato rocoso y las aguas freáticas. Al mismo tiempo, queda establecida con claridad la distribución de potencia y profundidad.

Con el Mapa Geotécnico generado, el proyectista tiene posibilidades de evaluar el terreno y el promotor de vislumbrar los condicionantes de la parcela. Así mismo, sirve para rea- lizar en primera instancia, una estimación económica de la cimentación.

Con carácter demostrativo, se han representado los planos correspondientes al estrato rocoso, mediante las curvas de igual profundidad, de igual espesor, así como la representación tridimensional de las curvas de igual profundidad. Finalmente, nos gustaría destacar que se ha llegado al rechazo en prácticamente la mitad de los sondeos.

Como líneas de trabajo futuras, se podría estudiar la presencia del nivel freático en la ciudad de Badajoz en función de las coordenadas espaciales y de la estación meteorológica, así como analizar los fenómenos de expansividad presentes en la misma.

\section{CONSIDERACIONES ÉTICAS}

El trabajo presentado forma parte de la tesis doctoral de Francisco Hipólito-Ojalvo, citada en la bibliografía.

\section{AGRADECIMIENTOS}

A los autores les gustaría agradecer a las compañías: LYCCSA y ELABOREX por proporcionar los resultados experimentales correspondientes a los estudios geotécnicos realizados entre 1990 y 2010. Y a cuantas personas han intervenido en la manipulación de los 459 sondeos.

\section{REFERENCIAS}

(1) Yilmaz, I. (2008). A case study for mapping of spatial distribution of free surface heave in alluvial soils (Yalova, Turkey) by using GIS software. Computers \& Geosciences, 34(8): 993-1004. https://doi.org/10.1016/j.cageo.2007.06.008

(2) You, Z., Fu, H. y Shi, J. (2018). Design-by-analogy: A characteristic tree method for geotechnical engineering. Automation in Construction, 87: 13-21. https://doi.org/10.1016/j.autcon.2017.12.008

(3) El May, M., Dlala, M. y Chenini, I. (2010). Urban geological mapping: Geotechnical data analysis for rational development planning. Engineering Geology, 116(1): 129-138. https://doi.org/10.1016/j.enggeo.2010.08.002

(4) Wan-Mohamad, W.N.S. y Abdul-Ghani, A.N. (2011). The Use of Geographic Information System (GIS) for Geotechnical Data Processing and Presentation. Procedia Engineering, 20: 397-406. https://doi.org/10.1016/j.proeng.2011.11.182

(5) Labib, M. y Nashed, A. (2013). GIS and geotechnical mapping of expansive soil in Toshka region. Ain Shams Engineering Journal, 4(3): 423-433. https://doi.org/10.1016/j.asej.2012.11.005

(6) Ma, J. y Cheng, J.C.P. (2016). Estimation of the building energy use intensity in the urban scale by integrating GIS and big data technology. Applied Energy, 183: 182-192. https://doi.org/10.1016/j.apenergy.2016.08.079

(7) Jahangiri, M., Ghaderi, R., Haghani, A. y Nematollahi, O. (2016). Finding the best locations for establishment of solarwind power stations in Middle-East using GIS: A review. Renewable and Sustainable Energy Reviews, 66: 38-52. https://doi.org/10.1016/j.rser.2016.07.069

(8) Sahoo, K., Hawkins, G.L., Yao, X.A., Samples, K. y Mani, S. (2016). GIS-based biomass assessment and supply logistics system for a sustainable biorefinery: A case study with cotton stalks in the Southeastern US. Applied Energy, 182: 260-273. https://doi.org/10.1016/j.apenergy.2016.08.114

(9) Vienneau, D., de Hoogh, K. y Briggs, D. (2009). A GIS-based method for modelling air pollution exposures across Europe. Science of The Total Environment, 408(2): 255-266. https://doi.org/10.1016/j.scitotenv.2009.09.048

(10) Apparicio, P., Carrier, M., Gelb, J., Séguin, A.-M. y Kingham, S. (2016). Cyclists' exposure to air pollution and road traffic noise in central city neighbourhoods of Montreal. Journal of Transport Geography, 57: 63-69. https://doi. org/10.1016/j.jtrangeo.2016.09.014

(11) Keshavarzi, B., Ebrahimi, P. y Moore, F. (2015). A GIS-based approach for detecting pollution sources and bioavailability of metals in coastal and marine sediments of Chabahar Bay, SE Iran. Chemie Der Erde - Geochemistry, 75(2): $185-195$. https://doi.org/10.1016/j.chemer.2014.11.003

(12) Chau, V.N., Holland, J., Cassells, S. y Tuohy, M. (2013). Using GIS to map impacts upon agriculture from extreme floods in Vietnam. Applied Geography, 41: 65-74. https://doi.org/10.1016/J.APGEOG.2013.03.014

(13) Jelokhani-Niaraki, M., Sadeghi-Niaraki, A. y Choi, S.-M. (2018). Semantic interoperability of GIS and MCDA tools for environmental assessment and decision making. Environmental Modelling \& Software, 100: 104-122. https://doi. org/10.1016/j.envsoft.2017.11.011

(14) Wang, Y., Huynh, G. y Williamson, C. (2013). Integration of Google Maps/Earth with microscale meteorology models and data visualization. Computers \& Geosciences, 61: 23-31. https://doi.org/10.1016/j.cageo.2013.07.016 
(15) Buchori, I., Sugiri, A., Mussadun, M., Wadley, D., Liu, Y., Pramitasari, A. y Pamungkas, I.T.D. (2018). A predictive model to assess spatial planning in addressing hydro-meteorological hazards: A case study of Semarang City, Indonesia. International Journal of Disaster Risk Reduction, 27: 415-426. https://doi.org/10.1016/j.ijdrr.2017.11.003

(16) Jiménez, M., García-Fernández, M., Zonno, G. y Cella, F. (2000). Mapping soil effects in Barcelona, Spain, through an integrated GIS environment. Soil Dynamics and Earthquake Engineering, 19(4): 289-301. https://doi.org/10.1016/ So267-7261(00)00007-5

(17) Francioni, M., Salvini, R., Stead, D., Giovannini, R., Riccucci, S., Vanneschi, C. y Gullì, D. (2015). An integrated remote sensing-GIS approach for the analysis of an open pit in the Carrara marble district, Italy: Slope stability assessment through kinematic and numerical methods. Computers and Geotechnics, 67: 46-63. https://doi.org/10.1016/j.compgeo.2015.02.009

(18) Thum, L. y De Paoli, R. (2015). 2D and 3D GIS-based geological and geomechanical survey during tunnel excavation. Engineering Geology, 192: 19-25. https://doi.org/10.1016/j.enggeo.2015.03.013

(19) Shen, H., Klapperich, H., Abbas, S.M. y Ibrahim, A. (2012). Slope stability analysis based on the integration of GIS and numerical simulation. Automation in Construction, 26: 46-53. https://doi.org/10.1016/j.autcon.2012.04.016

(20) Hernández del Pozo, J.C. (1998). Análisis metodológico de la cartografía geotécnica urbana, aplicación a la ciudad de Granada (Tesis Doctoral). Universidad de Granada.

(21) Vázquez Carretero, N.J. (2001). Cálculo de la subsidencia unidimensional debida a los descensos del nivel piezométrico. Aplicación al casco urbano de Murcia ya los efectos sobre sus edificios (Tesis Doctoral). Universidad de Sevilla.

(22) Jaramillo, A., Huete, R., Rodríguez, C., Suarez, L. y Sanz, V. (2005). Anejo 1.2. Mapa geotécnico de la ciudad de Sevilla. In Protocolo de Inspección Técnica de Edificaciones. Sevilla: Colegio Oficial de Arquitectos de Sevilla.

(23) Hernández, J.C., Ocete, I., González, J. y Hernández, J.C. (2006). Las cimentaciones en la ciudad de Melilla. Melilla: Consejería de Fomento de la Junta de Andalucía.

(24) INE. (2018). Censo municipal. Descargado 11 de mayo de 2018 desde http://www.ine.es/jaxiT3/Datos.htm?t=2911

(25) Ayuntamiento de Badajoz. (2014). Plan estratégico de la ciudad de Badajoz.

(26) AENOR. (1994a). UNE 103103:1994 Determinación del limite liquido de un suelo por el método del aparato de casagrande.

(27) AENOR. (1995). UNE 103 101:1995 Análisis granulométrico de suelos por tamizado.

(28) AENOR. (1993). UNE 103400:1993 Ensayo de rotura a compresión simple en probetas de suelo.

(29) AENOR. (2006b). UNE-EN ISO 22476-3:2006 Investigación y ensayos geotécnicos. Ensayos de campo. Parte 3: Ensayo de penetración estándar.

(30) AENOR. (1994b). UNE 103801:1994 Geotecnia. Prueba de penetración dinámica superpesada.

(31) AENOR. (2006a). UNE-EN ISO 22476-2:2005 Investigación y ensayos geotécnicos. Ensayos de campo. Parte 2: Ensayo de penetración dinámica.

(32) Hipólito-Ojalvo, F. (2016) Evaluación experimental del subsuelo y desarrollo de la cartografía geotécnica de Badajoz utilizando sistemas de información geográfica para reducir los riesgos de patologías estructurales (Tesis Doctoral). Universidad de Oviedo.

(33) Pintos, S. (2012). Predicción geoestadística: kriging simple, ordinario y con tendencia. Editorial Universidad de Zulia.

(34) Davies, T.G. y Banerjee, P.K. (1978). The displacement field due to a point load at the interface of a two layer elastic halfspace. Geotechnique, 28(1): 43-56. https://doi.org/10.168o/geot.1978.28.1.43

(35) Bosque Sendra, J. (1992). Sistemas de información geográfica. Madrid: Rialp.

(36) Matheron, G. (1962). Traité de géostatistique appliquée (Vol. 1 y 2). Ediciones Technip.

(37) España. (2006). Real Decreto 314/2006, de 17 de marzo, por el que se aprueba el Código Técnico de la Edificación. Boletín Oficial Del Estado, (74, 28 de Marzo 2006): 11816-11831. 